\title{
EMITTANCE ANALYSIS OF BEAM PROPAGATION IN LOW PRESSURE AIR
}

\author{
J. R. Smith, W. Namkung, R. F. Schneider, and M. J. Rhee ${ }^{a}$ \\ Naval Surface Weapons Center, White Oak \\ Silver Spring, MD 20903-5000
}

\section{Abstract}

Relativistic electron beam propagation in a conducting tube filled with low pressure air is investigated. Filling pressures from 20 to $320 \mathrm{mTorr}$ were used. For all of the pressures in this range a significant portion of the beam current was transported over a $1 \mathrm{~m}$ tube length. Detailed emittance analysis characterizing beam propagation in the low pressure air is presented.

\section{Ion Focusing Regime Propagation}

Ion focusing regime propagation (IFR) involves relativistic electron beam propagation in gas at low (generally subTorr) pressures. The beam is injected into the low pressure gas which is ionized by electron impact ionization. Simultaneously as a plasma channel is created, at the beam front secondary electrons are ejected by the heavier relativistic beam electrons resulting in a plasma channel with a net positive charge. It is required that these secondary electrons do not create additional ionization as they are ejected. The ions in the plasma channel produce a force which opposes that of the beam's space charge hence the terminology ion focusing regime

propagation. Additional to these electrostatic forces is the self magnetic pinching force. Radial force balance may be obtained in the IFR allowing stable propagation. A more complete description of IFR propagation may be found in the literature. 1,2 Some comments on the parameter regime required for IFR propagation are appropriate. There must be a sufficient ion density for a significant focusing force. This sets a lower bound on the fractional charge neutralization $f\left(\equiv n_{i} / n_{b}\right)$ such that $f>$ $1 / \gamma^{2}$. The upper bound on $f(i . e . f<1)$ is due to the requirement that the beam density must exceed the electron plasma density to avoid the electron-electron two stream instability. These requirements on $f$ may be rewritten as $n_{i}<n_{b}<\gamma^{2} n_{i}$. For readily obtained ion densities, and the additional requirement that the ejected secondary electrons do not cause further ionization, this translates into a beam density of approximately $10^{11} / \mathrm{cm}^{3}$. Typically this means beam currents are in the kiloampere range. Beam energy must be sufficiently relativistic such that the beam electrons at the the beam front are heavy enough to efficiently eject secondary electrons.

In Ref. 2 an estimate for the critical pressure for IFR propagation is given. For nitrogen gas or air the pressure is given by

$$
p<\left(\frac{I_{B}}{10 k A}\right) \frac{1}{a_{0}} \text { Tor }-c m
$$

a) Permanent address, University of Maryland, College Park MD 20742. where $a_{0}$ is the beam radius. For the $3 \mathrm{kA}, 1 \mathrm{~cm}$ radius beam used for this work, the pressure is less than 300 mTorr for pure IFR propagation.

\section{Root-Mean-Square Emittance}

Beam emittance is a function of beam radius and transverse particle motion. The transverse motion of beam particles consists of a random motion due to the temperature (inherent in the beam source, scattering by anode foils, etc.) and/or a nonrandom radial motion due to beam divergence. Generally a high quality beam is characterized hy a low emittance value. Beams with low emittance are particularly desirable where efficient propagation is desired. The following is a short background summary of the rms emittance analysis as applied to our beam. Two assumptions are made: (1) an axisymmetric beam, and (2) a thermal distribution (Maxwellian). Using these assumptions the distribution function in transverse trace space may be written as

$$
\begin{aligned}
& f_{4}\left(x, y, x^{\prime}, y^{\prime}\right)=g(x, y) \text { exp }\left\{-\left[\left(x^{\prime}-\bar{x}^{\prime}\right)^{2}\right.\right. \\
& \left.\left.+\left(y^{\prime}-\bar{y}^{\prime}\right)^{2}\right] / 2 \sigma^{2}(x, y)\right\}
\end{aligned}
$$

where $x^{\prime}=d x / d z$ and $y^{\prime}=d y / d z$ are the gradients of the particle trajectory, $g(x, y)$ is the spatial dependence of the distribution function, $\sigma$ is the rms value of the random trajectory angles,

and $\bar{x}^{\prime}$ and $\bar{y}^{\prime}$ are the mean angles as functions of $x$ and $y$. The rms emittamce is given by

$$
\varepsilon_{\text {rms }}=4\left[\left\langle x^{2}\right\rangle\left\langle x^{\prime 2}\right\rangle-\left\langle x x^{\prime}\right\rangle^{2}\right]^{1 / 2}
$$

where < > denotes the average over the four dimensional trace space distribution function. The emittance meter used in this work consists of a series of slits oriented parallel to the $y$ axis. An electron beam sensitive film is used as a detector to record the profile of the sheet beamlets. This film is scanned with a microdensitometer using a small aperture (pinhole) so that only particles impinging on the detector at the $y=0$ axis are recorded. The beam is first sampled through the slits of the emittance meter, and then through the pinhole of the microdensitometer, therefore this method of analysis is equivalent to the slit-pinhole emittance meter. By analysis of the microdensitometer scan we determine

$g(x, y=0), \sigma(x, y=0)$, and $\bar{x}^{\prime}(x, y=0)$. Using the assumptions made, $f_{4}\left(x, y, x^{\prime}, y^{\prime}\right)$ is determined over all space, and the rms emittance is calculated numerically using Eq. (3). 


\section{Experiment}

The Transbeam accelerator at NSWC was used for the experimental investigation described in this paper. Transbeam uses a coaxial pulse forming line which is charged by a high voltage transformer. The transformer has a 1:41 turn ratio and is similar to the design of Rohwein. ${ }^{3}$ A $30 \mathrm{~kJ}$, two stage capacitor bank connected to the primary turn is used to pulse the transformer. The electron beam produced by Transbeam has $700 \mathrm{keV}$ energy and a pulse duration of $100 \mathrm{~ns}$. The diode electrodes consist of a $7.5 \mathrm{~cm}$ diameter carbon cathode and a $0.6 \mathrm{mil}$ thick titanium anode foil. For the IFR propagation work a high quality beam with a beam current of a few kiloamperes is desired. Therefore, immediately downstream of the anode foil a range thick carbon disk with a $2 \mathrm{~cm}$ diameter aperture is placed. This carbon mask allows only electrons emitted from the central portion of the planar cathode to be injected into the drift tube. Also it reduces the beam current from approximately 66 $\mathrm{kA}$ in the diode to 2-3 kA at the drift tube entrance. The experimental setup is shown in Fig. 1.

The drift tube was either a $10 \mathrm{~cm}$ diameter stainless steel tube, or a $10 \mathrm{~cm}$ diameter acrylic tube with a copper screen insert which allowed for photographic diagnostics. The tube length was varied according to the experimental setup. The experiment was performed in vacuum with the drift tube at 0.01 mTorr and with room air admitted at pressures of $20,40,80,160$, and $320 \mathrm{mTorr}$.

Side on open shutter photography was employed to observe the gross behavior of beam propagation. For all pressures there was reasonably good propagation as observed from the gas light, although lateral displacement was present in some shots. More or less, the beam radius retained its value at injection. At pressures of 160 and 320 mTorr the beam exhibited enhanced focusing at an axial distance approximately equal to 5 beam radii.

RADIACHROMIC FILM
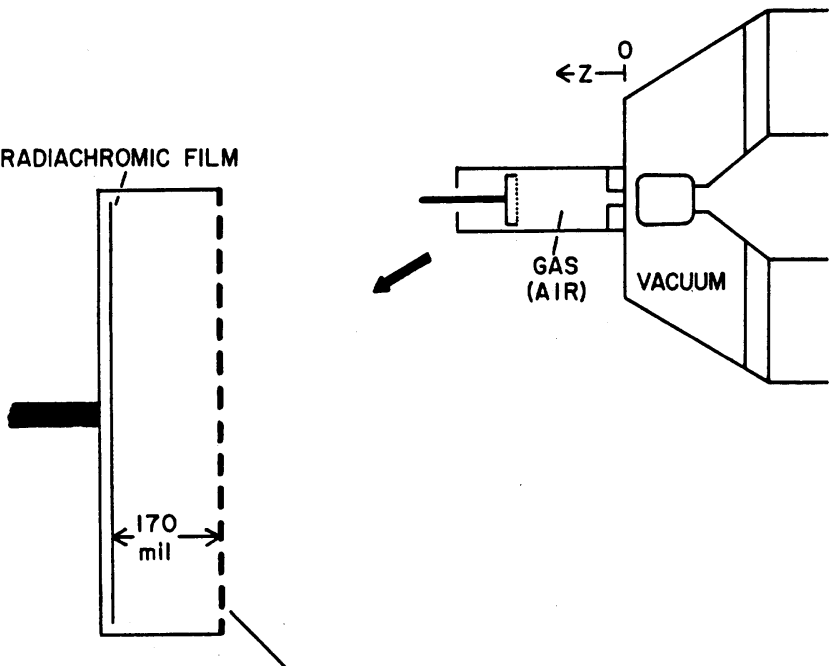

$16 \times 195 \mathrm{mil}$ Ta BARS

20 mII SLIT WIDTH
Diode voltage and current waveforms were very reproducible from shot to shot. The beam current entering the drift tube was approximately $3 \mathrm{kA}$ as measured by a Rogowski coil or a Faraday cup. This represents $5 \%$ of the diode current. In order to determine transported beam current a $2 \mathrm{~cm}$ diameter Faraday cup was positioned $84 \mathrm{~cm}$ downstream of the anode foil. Transported beam current ranged from 1 to $2 \mathrm{kA}$ for the various gas pressures, with no particular trend evident. We emphasize that this Faraday cup has a $2 \mathrm{~cm}$ diameter and therefore only monitors the portion of beam that remains strictly on axis.

Details of the emittance mask are also shown in Fig. 1. A 2-mil thick radiachromic film was used as the detector. ${ }^{4}$ After exposure to one shot, the film was scanned with a microdensitometer using a resolution spot size of $0.9 \times 0.02 \mathrm{~mm}$. Results at different axial positions and pressures are given in Fig. 2. The rms emittance was calculated by the method previously described and the results appear in Table I. The injected beam is characterized by an emittance of $0.55 \mathrm{~cm}$-rad. This value increases by a factor of 1.5 to 2 after a short propagation distance in gas, with smaller fluctuations occuring during later propagation. The emittance increase is attributed to scattering of the beam by the gas. ${ }^{5}$ Emittance contour plots for two shots are given in Fig. 3. The skew contours of the vacuum shot show the effect of space charge divergence, and the upright contours of the 80 mTorr shot show the effect of ion focusing.

\section{Acknowledgements}

This work was supported by the Independent Research Fund at the Naval Surface Weapons Center.

Table I. Emittance values characterizing beam propagation in low pressure air.

\begin{tabular}{c|ccccc}
\hline$z(\mathrm{~cm})$ & 20 & 40 & 80 & 160 & 320 \\
\hline 40 & 0.97 & 1.14 & 0.89 & 0.88 & 0.80 \\
30 & 0.95 & 0.92 & 0.95 & 0.80 & 0.69 \\
20 & 0.80 & 0.90 & 0.86 & 0.86 & 0.85 \\
10 & 0.82 & 1.06 & 0.76 & 0.68 & 0.67 \\
\hline
\end{tabular}

Fig. 1. Experimental configuration. 


\section{References}

1. R. J. Briggs, "A Simple Model of Beam Transport in Low-Pressure Ion Focused Regimes," Lawrence Livermore National Laboratory, Report UCID-19187, September 1981.

2. R. J. Briggs and S. Yu, "Modeling Beam Front Dynamics at Low Gas Pressures," Lawrence Livermore National Laboratory Report UCID-19399, May 1982.

3. G. J. Rohwein, "A Three Megavolt Transformer for PFL Pulse Charging," IEEE Trans. Nucl. Sci. NS-26, 4211 (1979).

4. A. D. Kantz and K. C. Humpherys, "Quality Assurance for Radaition Processing," Radiat. Phys. Chem. 14, 575 (1979).

5. J. D. Lawson, The Physics of Charged Particle Beams, (Oxford University Press, 1977).

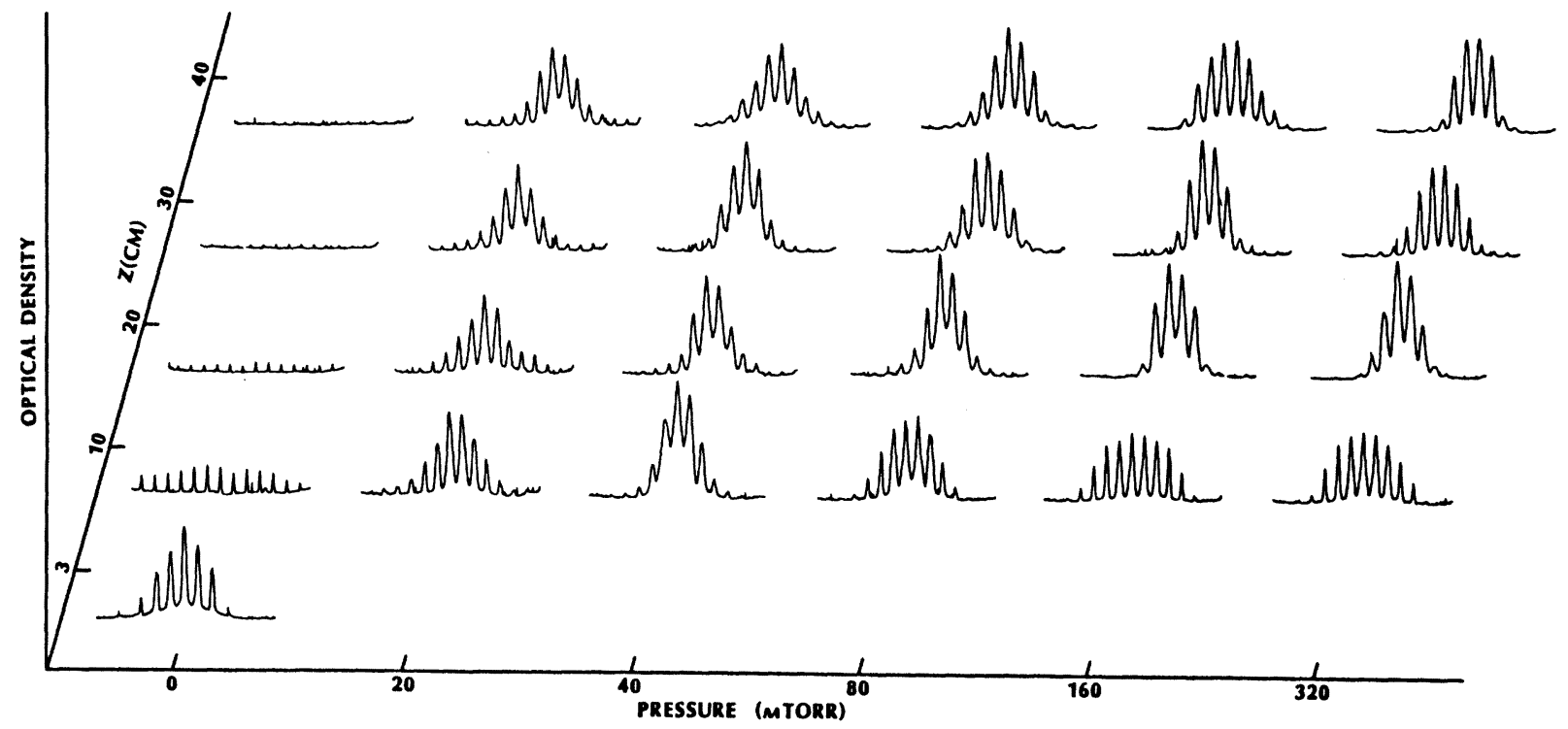

Fig. 2 - Microdensitometer scans of radiachromic film at different axial positions $z$ and pressures.

(a)

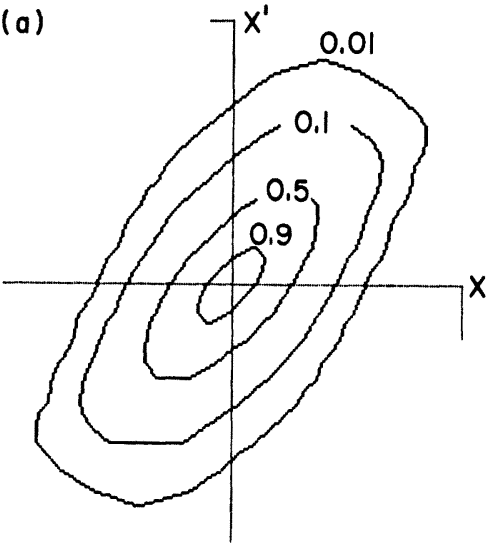

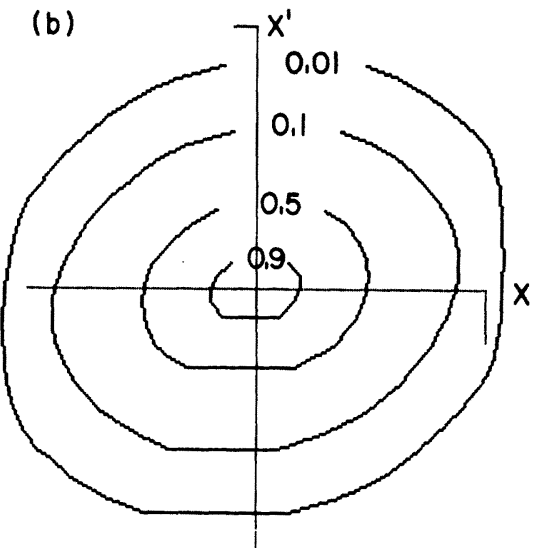

Fig. 3 - Emittance plots showing equidensity contours. Case (a) represents $z=3 \mathrm{~cm}, p=0 \mathrm{mTorr}$, and case (b) is for $z=30 \mathrm{~cm}, p=80$ mTorr. 